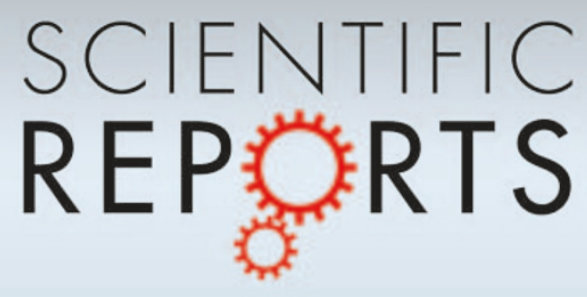

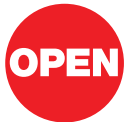

SUBJECT AREAS:

NANOTECHNOLOGY

ELECTRONIC MATERIALS AND

DEVICES

SYNTHESIS

APPLIED PHYSICS

Received

16 November 2011

Accepted

23 April 2012

Published

4 May 2012

Correspondence and requests for materials should be addressed to

H.W.Z.

(hongweizhu@

tsinghua.edu.cn)

\section{Multifunctional graphene woven fabrics}

\author{
Xiao Li', Pengzhan Sun', Lili Fan', Miao Zhu' ', , Kunlin Wang ', Minlin Zhong' ', Jinquan Wei', Dehai Wu', \\ Yao Cheng ${ }^{2,3}$ \& Hongwei Zhu ${ }^{1,2}$
}

\begin{abstract}
'Department of Mechanical Engineering, Key Laboratory for Advanced Manufacturing by Materials Processing Technology, Tsinghua University, Beijing 100084, PR China, ${ }^{2}$ Center for Nano and Micro Mechanics (CNMM), Tsinghua University, Beijing 100084, PR China, ${ }^{3}$ Department of Engineering Physics, Tsinghua University, Beijing 100084, PR China.
\end{abstract}

Tailoring and assembling graphene into functional macrostructures with well-defined configuration are key for many promising applications. We report on a graphene-based woven fabric (GWF) prepared by interlacing two sets of graphene micron-ribbons where the ribbons pass each other essentially at right angles. By using a woven copper mesh as the template, the GWF grown from chemical vapour deposition retains the network configuration of the copper mesh. Embedded into polymer matrices, it has significant flexibility and strength gains compared with CVD grown graphene films. The GWFs display both good dimensional stability in both the warp and the weft directions and the combination of film transparency and conductivity could be optimized by tuning the ribbon packing density. The GWF creates a platform to integrate a large variety of applications, e.g., composites, strain sensors and solar cells, by taking advantages of the special structure and properties of graphene.

$\mathrm{T}$ he creation and modulation of the nanomaterials' structure and function are of great scientific interest and importance. Nanomaterials (especially one-dimensional, 1D) are important building blocks for nanoscale electronics ${ }^{1,2}$, as they can function as miniaturized devices as well as electrical interconnects ${ }^{3,4}$. A typical example of macrostructures from self-assembly is metamaterial ${ }^{5}$. Metamaterials are artificial materials engineered to have unique properties gained from structure rather than composition, using small inhomogeneities to create effective macroscopic behavior ${ }^{6,7}$. Another example is woven or non-woven fabrics of nanomaterials, including organic electronic fibres for logic circuits ${ }^{8}$, oxide nanowires for photoswitches and memories ${ }^{9}$, electrospun nanofibers for composites ${ }^{10}$, "smart" textiles for sensors and displays ${ }^{11}$, woven electrodes for photovoltaics ${ }^{12}$, self-organized silicon nanowire network for highly-integrated crossbar nanocircuits ${ }^{13}$.

Regarding carbon nanomaterials, carbon nanotube (CNT) 1D long fibers, two-dimensional (2D) thin films and three dimensional (3D) arrays and sponges have been widely investigated ${ }^{14}$. Cross-stacked CNT films have been used for transparent and stretchable conductors ${ }^{15}$. Recently, graphene macrostructures have also attracted a lot of attention, e.g., foam ${ }^{16,17}$, mesh ${ }^{18}$, pattern ${ }^{19}$, ribbons ${ }^{20}$, which take the advantages of the outstanding properties of graphene to make full use of its macroscale assembly.

In this work, we report a simple route to realize a graphene-based woven fabric (GWF), that is assembled by intersecting graphene micron-ribbons (GMRs). To our knowledge, this is the first report on the woven fabric structure made from aligned GMRs. The structural and characteristic features of our GWFs are: (i) It possesses high structural integrity and much better mechanical performance compared with polycrystalline graphene films; (ii) In contrast to monolayer graphene, it has good gas/liquid permeability (due to the presence of micron holes); (iii) Upon external loading, it shows an interesting exponential resistive response which is favourable for high sensitivity strain sensing. Based on the unique structural features and properties, we demonstrate the following applications of our GWFs: (i) GWF/polymer composites, which are well aligned GMRs embedded in polydimethylsiloxane (PDMS) to produce 2D network with multi-joint conductive channels, or deposited on PDMS as strain sensors. (ii) GWF/semiconductor solar cells, in which the GWF functions as the transparent electrode in Schottky junction to provide enhanced photovoltaic conversion. Most importantly, the periodic voids of GWFs can be filled with other functional materials, or served as a permeable membrane of electrolytes for solar cell applications.

\section{Results}

Structure and morphology of the GWF. GWFs were grown by atmospheric CVD using copper meshes as substrates (see Methods section). Fig. 1a shows the three main steps used in the fabrication of GWFs: (i) CVD growth of graphene on copper mesh, (ii) removal of copper wires with $\mathrm{FeCl}_{3} / \mathrm{HCl}$ aqueous solution, and (iii) collapse of graphene to form double layered GMRs. The CVD grown GWF retains the network configuration of the copper mesh. For the cases described here, the copper mesh consists of copper wires ( $\sim 60 \mu \mathrm{m}$ in diameter), 

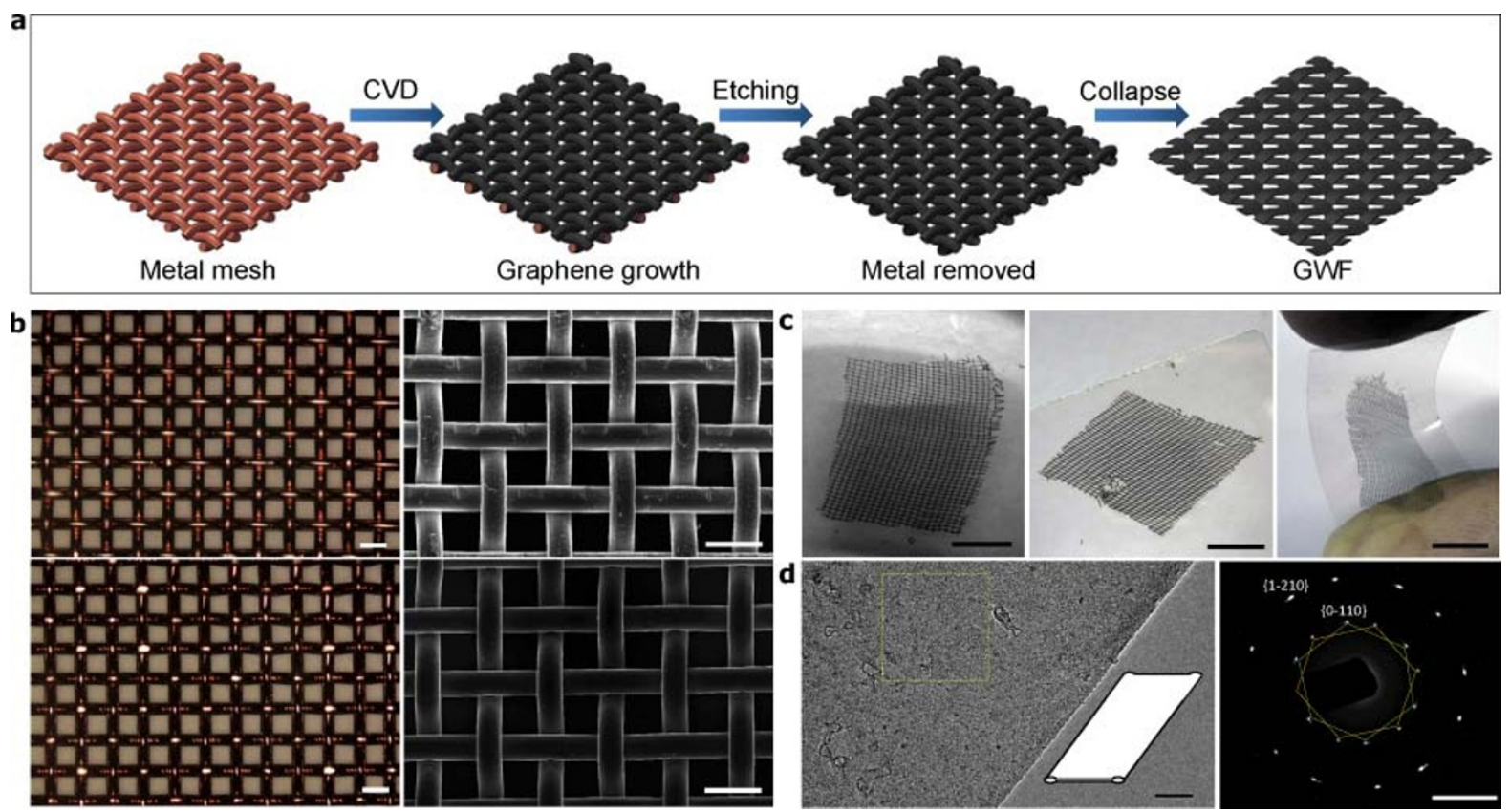

Figure 1 Fabrication of GWFs by CVD using copper wire meshes as substrates. (a) Schematic of steps for GWF preparation. (b) Macroscopic optical images (left), top-view SEM images (right) of copper meshes before (top) and after (bottom) graphene growth. Scale bars, $200 \mu \mathrm{m}$. (c) Optical images of GWF films floating on water and deposited on glass and PET. Scale bars, $5 \mathrm{~mm}$. (d) TEM image of a GMR and selected area electron diffraction pattern from the region marked with a yellow box. Scale bars, $50 \mathrm{~nm}$ (left), 5 (1/nm) (right).

arranged in a crisscross pattern. Fig. 1b shows the optical and planview scanning electron microscope (SEM) images of a copper mesh before and after graphene growth. In the next step, the GWFs can be collected from the liquid surface with desired target substrates. The details are described in the Methods section. Fig. 1c shows the asobtained GWF films floating on water and deposited on glass and PET, clearly demonstrating the well-aligned arrays of GMR lines, with width and spacing of $\sim 100 \mu \mathrm{m}$ and $\sim 150 \mu \mathrm{m}$, respectively. Every piece of the GWF is an integration of warp and weft GMRs through intersection. The enhanced contrast in Fig. 1c shows that the GMRs are clearly distinguished from the surrounding non-deposited areas, revealing the grid structure, thus demonstrating the precisely controlled dimension of the GWF pattern.

Fig. 1d shows the TEM image of a GMR and the corresponding electron diffraction pattern. TEM studies reveal that the GMR surface is clean and homogeneous. The electron diffraction pattern shows the typical six-fold symmetry expected for graphene and graphite. The diffraction pattern shows two sets of the hexagonal spots with $26^{\circ}$ rotation between them. This occurs because the electron beam simultaneously probes the front and back layers of the GMR, revealing its overlapping structure (as shown in the inset). Diffraction spots are labeled using Miller-Bravais ( $h k i l)$ indices. The analysis of the diffraction intensity ratio $I_{\{1-210\}} / I_{\{0-110\}}(\sim 0.7)$ reveals that the graphene is in fact few-layered ${ }^{21}$. Monolayer graphene has been grown on copper mesh; however, they are too fragile to form a monolithic structure upon copper etching. Raman spectra of the regions marked in Fig. S1 clearly show the evidences of bilayer and few-layer graphene, which are confirmed by the 2D-band $\left(2700 \mathrm{~cm}^{-1}\right)$ with a full width at half maximum of $40 \sim 60 \mathrm{~cm}^{-1}$ and by the moderate ratio $(0.6 \sim 1.6)$ of the $2 \mathrm{D}, \mathrm{G}$ peak intensities ${ }^{22}$, respectively. The weak peaks of D-band in the spectra reveal the presence of imperfections (e.g., surface defects, wrinkles, edges, grain boundaries) in the GWFs.

For comparison, nickel meshes were also used to grow GWFs by a non-equilibrium surface segregation process based on a carburization/decarburization mechanism. The multilayer nature of CVD graphene formed on nickel greatly delayed the etching process and left residual nickel-carbon compound cores inside GMRs. As shown in
Fig. S2, the so-obtained GWFs possess higher structural integrity compared with GWFs grown on copper. To demonstrate its mechanical strength, the GWF was collected by a quartz O-ring and after drying it can be self-supported.

Fig. 2 provides a large-area optical image (Fig. 2a) and magnified top views (Fig. 2b) of regions of a representative sample deposited on a silicon wafer. It can be clearly seen that the so-obtained GWF has a planar structure produced by interlacing two sets of GMRs which pass each other essentially at right angles, forming a self-locked planar fibrous system. Less transparent areas in Fig. $2 \mathrm{~b}$ can be attributed to the folding and overlap of a single layer or the overlap of multiple layers, and the darkest areas result from crumpled regions. The mild etching process keeps the integrity and configuration of the textile structure of the copper mesh, as is evident by the crosssectional view in Fig. 2c. The lower and upper parts of overlapped GMRs at the region of cross linking would adhere and stick together.

GWF/polymer composites. We then show the fabrication of GWF/ polymer composite films (bonding with inert, transparent polymer, e.g., PDMS). To obtain the GWF/PDMS composite mesh, before etching away the copper skeleton by $\mathrm{FeCl}_{3} / \mathrm{HCl}$ solution, a thin layer of PDMS with controlled thickness was deposited on the surfaces of the graphene-coated copper wires, followed by crosslinking and curing the PDMS as a support to prevent graphene networks from collapsing during copper etching. As shown in Fig. 2d, real free-standing GWF/PDMS composite films can be obtained after copper etching. In spite of the thin layer of PDMS coating, this composite structure still has good optical and electrical properties. As revealed in the insets of Fig. 2d, the free-standing GWF/PDMS composite film can be twisted by tweezers without fracture. The use of the PDMS support layer was critical for preparing the free-standing GWF composite film. This method can keep the 3D structure of GWFs (see bottom inset of Fig. 2d). As further revealed in Fig. S3, the composite film is woven by interconnected graphene/PDMS micron-tubes, forming a multijoint channel system. During CVD growth, two interconnected copper wires will melt and join together at the crossing point at $1000^{\circ} \mathrm{C}$, resulting in a monolithic structure of the copper mesh. 


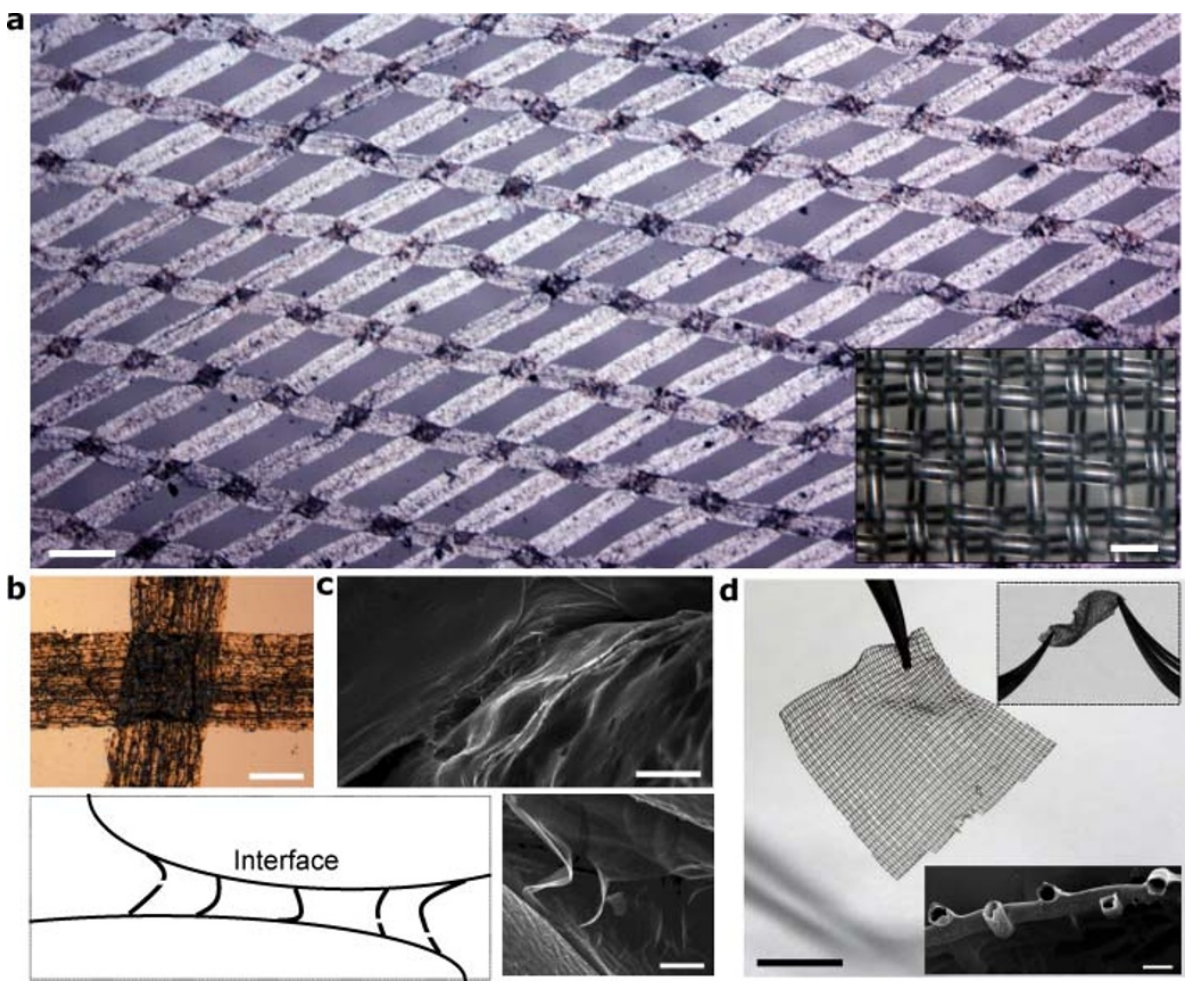

Figure 2 Large-area, CVD grown GWFs in supported and free-standing states. (a) Large-area optical image of a representative region of a GWF film. Scale bar, $100 \mu \mathrm{m}$. Inset shows the optical image of a nylon woven fabric for comparison. Scale bar, $200 \mu \mathrm{m}$. (b) Top-view optical image (Scale bar, $50 \mu \mathrm{m}$ ) and (c) High magnification SEM images of cross-sectional views of the interlacing points of GMRs. Scale bars, $5 \mu \mathrm{m}$ (top), $1 \mu \mathrm{m}$ (bottom). (d) Optical images of flexible GWF/PDMS composite films. Scale bar, $5 \mathrm{~mm}$. The top inset shows the twisted GWF film by tweezers. The bottom inset shows the cross-section view SEM image of the composite film. Scale bar, $100 \mu \mathrm{m}$.

Carbon was introduced by decomposing methane, and graphene was then grown on the surface of the interconnected copper wires. In the resulting composite (Fig. S4), graphene serves as a lining of the PDMS hollow structure and could be used as protective coating, reinforcement phase, electrical and thermal conducting channels in the composites.

To form fully filled GWF/PDMS composites (Fig. S3a and Fig. S4), a large amount of PDMS has been applied. The transparency of the composite film is high as the GWF is semi-transparent, and the GWF mesh was planarized and stabilized by filling the spacing between GMRs with PDMS.

Tunable morphology and optoelectronic properties. The diameter of the copper wire and the mesh density directly determine the structure of GWFs. The three frames of Fig. 3a show optical images of three GWFs produced using copper meshes woven with $100 \mu \mathrm{m}$ thick wires of different packing density. The corresponding representative small-area SEM views of these three samples are shown in Fig. 3b. Visual inspection of the samples and highresolution images collected from them indicate excellent spatial uniformity and low defect density. The packing density of GMRs is also tunable by post manipulation (Fig. S5), for instance, controlling the liquid flow during film transfer.

Due to the unique configuration, the GWF becomes inherently strong (compared with polycrystalline graphene films) and conductive. The optoelectronic performance in terms of sheet resistance $\left(R_{\mathrm{s}}\right)$ and transparency of GWF strongly relies on the geometrical parameters, such as GMR width, spacing (related to graphene coverage density). As shown in Fig. 3c,d, the film transparency is varied owing to the cavity of the GWF. The $R_{\mathrm{s}}$ of $500 \sim 2500 \Omega / \mathrm{sq}$ and $200 \sim 1200 \Omega / \mathrm{sq}$ after $\mathrm{HNO}_{3}$ treatment at the corresponding transmittances of
$50 \% \sim 90 \%$ are obtained. The resistance values still cannot meet the criteria for transparent conducting applications that may replace the conventional indium tin oxide (ITO). The combined conductivity and transparency property of the GWF is also not advantageous due to its polycrystalline structure and the presence of surface wrinkles which will greatly reduce the conductivity and transparency of GMRs, in turn, GWFs. The conductivities of the GWFs are expected to be improved by synthesis process optimization ${ }^{23}$ and post acid doping $^{24}$.

GWF/PDMS hybrid films. GWFs were transferred on PDMS substrates to make GWF/PDMS hybrid films, and their mechanical properties (in tension) were studied. The interlacing (or crossing) points are the major locations where interactions between GMRs take place, through which the GWF forms an interlocked structure. We demonstrate that conductive GWF/PDMS hybrid films can act as electromechanical sensors under uniaxial tension. Tensile test accompanied by simultaneous electrical measurement shows a significant increase of resistance during the tensile cycle. The strain dependent resistance variations of GWF/PDMS films were investigated and summarized in Fig. 4a. As shown in the schematics (left panel), the $\mathrm{X}$ direction is defined as the direction of GMR lines and the XY direction is defined as the diagonal direction.

The electromechanical response differs along different directions and under different strains. The hybrid film shows certain flexibility and can be stretched within a relatively low strain range $(<5 \%)$ and the resistance returns to the initial value upon unloading (Fig. $4 \mathrm{a}$ ). Irreversible tensile strain and resistance changes occur at relatively high strain levels. The hybrid film shows a significant jump of resistance (by several orders of magnitude, up to $10^{5}$ times) when it was 
a

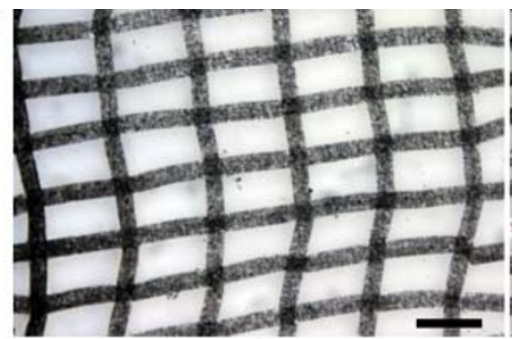

b
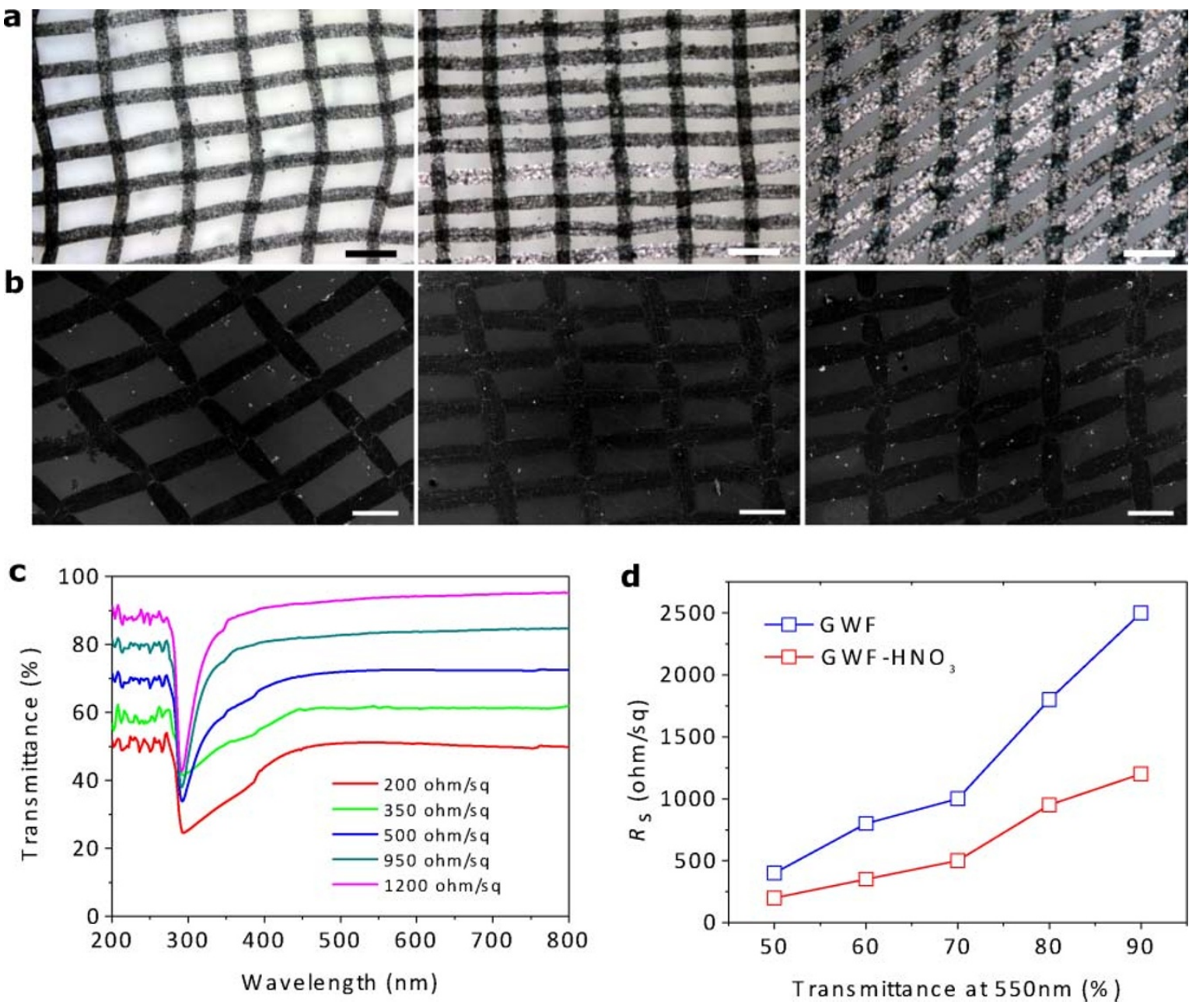

Figure 3 Macroscale GWFs with tunable GMR packing density. (a) Macroscopic optical images of three different GWFs (Scale bars, $300 \mu \mathrm{m}$ ) and (b) corresponding representative small-area SEM views of these three samples. Scale bars, $200 \mu \mathrm{m}$. (c) Ultraviolet-visible-near infrared transmission spectra and (d) transparency (at $550 \mathrm{~nm}$ ) versus sheet resistance plots.

stretched to a strain of $12 \%$ (Fig. $4 \mathrm{a}$ ), due to the fracture occurring along the boundaries within the polycrystalline GMRs (Fig. S6). When the hybrid film is returned to $0 \%$ strain, the resistance increases to $3 \sim 5$ times of its original value. In comparison, when stretched along the $\mathrm{XY}$ direction, the quadrilateral GMR network is self-adjusting. The stress applied on GMRs is smaller than that when the same strain is applied along X direction. If the PDMS substrate was pre-stretched before GWF deposition, the resistance variation becomes more gently. As shown in Fig. 4a, the resistance change is only 3 times when a pre-stretched sample was stretched to $10 \%$ along $\mathrm{XY}$ direction. Similar resistance change has been observed in several other graphene/PDMS ${ }^{25}$ and $\mathrm{CNT} / \mathrm{PDMS}^{26}$ composites under stretching. Our GWF/PDMS hybrid film shows reversible resistance change $(\Delta R)$ relative to the original value $\left(R_{0}\right)$ over many cycles and maintains a non-linear relationship versus strain in every cycle. Fig. $4 \mathrm{~b}$ shows the results at maximum stains of $2 \%$ and $5 \%$ for a GWF/PDMS sample along $X$ direction. The resistance shows an increase of 25 times under $2 \%$ strain and 230 times under 5\% strain. Based on this result, the GWF can not only be used as a strain sensor for composites, if high resolution and large image fields of view could be obtained, the resistance variation and optical diffraction from optical images might provide information for the deformation of the composite. As a demonstration, the GWF/PDMS hybrid film was further fixed to an index finger to perform as a stretchable sensor. The resistance was detected at different finger motion. As shown in Fig. $4 \mathrm{c}$, taking about $45^{\circ}$ as a step, the resistance of the hybrid film increases with finger bending, which is similar to the results in the stretching experiments.

GWF/Si Schottky solar cells. Alternative approaches to achieve transparent electrodes have investigated the use of with conductive nanostructures ${ }^{27}$ such as graphene, CNTs and metallic nanowires, or conductive polymers such as poly(3,4-ethylenedioxythiophene)/ poly(styrene sulfonic acid) (PEDOT:PSS). Though the GWF is not a good window electrode material for transparent conductor applications, the periodic voids can be filled with PEDOT, or served as permeable membrane of electrolytes for photovoltaic applications. Our techniques have provided transparency on the order of $70 \% \sim 80 \%$, with conductivities in the $1000 \Omega /$ sq range. GWF/Si solar cells were first fabricated from the GWF transparent electrodes and compared with similar devices fabricated using graphene film ${ }^{28}$. The solar cells with GWF electrodes outperformed devices having graphene transparent electrodes in efficiency $(\eta)$ $(>2 \%$ and up to $3.8 \%$, see Fig. S7). While these parameters are sufficient for some flexible electronics applications, the resistive losses remain too high for many scaled applications. The discontinuity of the GWF films also leads to other issues impacting device performance. As shown in Fig. 5a, the GWF-Si solar cell was doped by $\mathrm{HNO}_{3}$ fume. Acid infiltration of GWF networks boosts the cell efficiency by reducing the internal resistance to improve the fill factor (FF) and by forming photoelectrochemical units that enhance charge separation and transport ${ }^{29,30}$. In this way, the efficiency of 

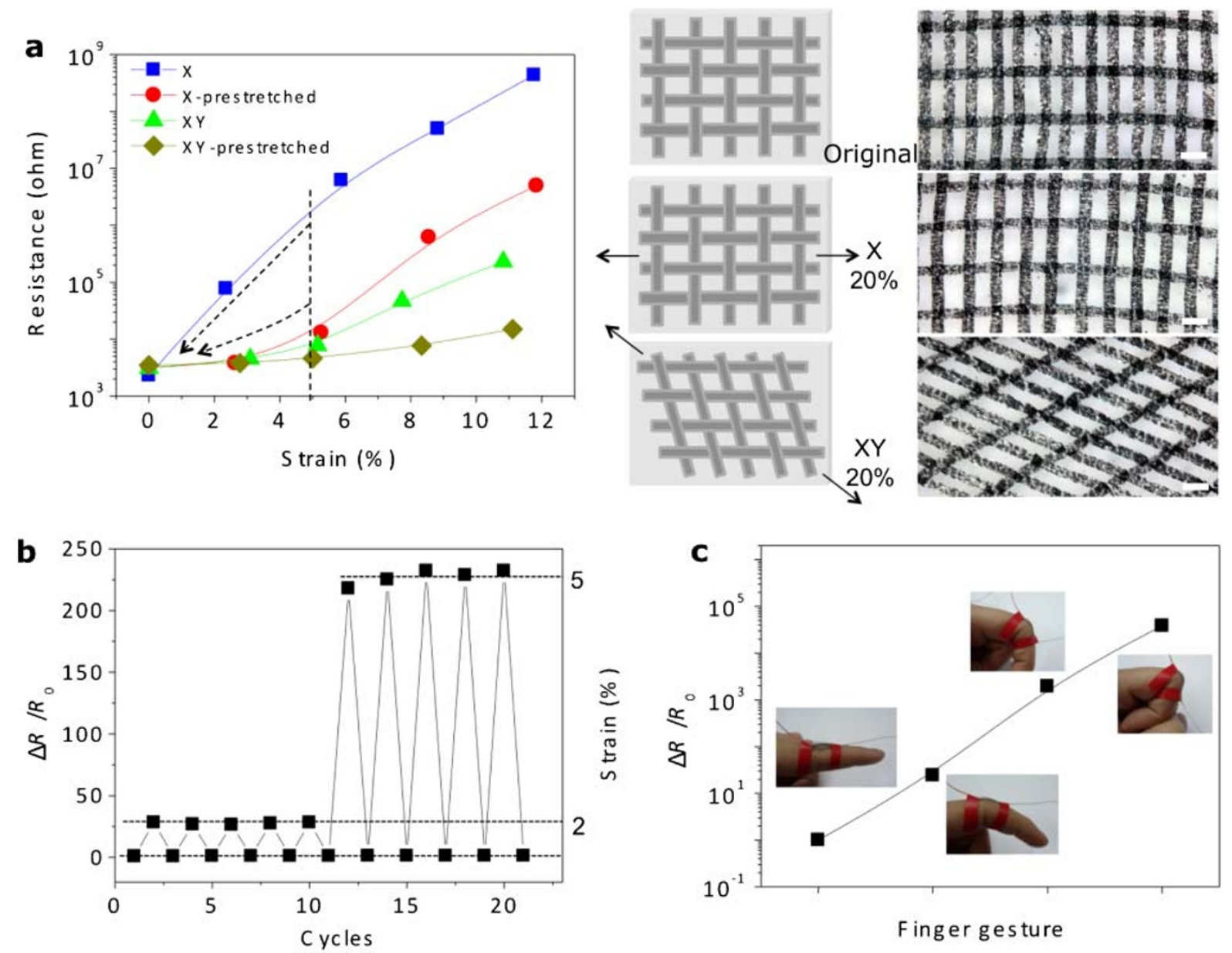

Figure $4 \mid$ GWF/polymer hybrid films. (a) Resistance-strain curves for GWF/PDMS hybrids along different directions. Inset shows the schematics and corresponding optical images. Scale bars, $300 \mu \mathrm{m}$. (b) Electromechanical properties of the GWF/PDMS films. Resistance change relative to the original value $\left(\Delta R / R_{0}\right)$ recorded for a number of cycles at tensile strains of $2 \%$ and $5 \%$. (c) Stretchable sensor fixed to a finger, and relative changes in resistance for finger motion. Insets show corresponding photographs.

solar cells could reach $6.1 \%$ (2-fold increase), with $V_{\mathrm{oc}}$ of $0.53 \mathrm{~V}$, short-circuit current density $\left(J_{\mathrm{sc}}\right)$ of $15.6 \mathrm{~mA} / \mathrm{cm}^{2}$, and fill factor (FF) of $58.4 \%$ at AM $1.5\left(80 \mathrm{~mW} / \mathrm{cm}^{2}\right)$.

Based on the GWF/Si solar cell, PEDOT was further added and filled in the cavity of the GWF. PEDOT was first spin coated and then annealed at $140^{\circ} \mathrm{C}$ for $15 \mathrm{~min}$, producing a GWF-PEDOT composite film. The introduction of PEDOT, commonly used as a buffer layer, facilitates the hole injection and extraction (Fig. 5b). The work function of GWF is $\sim 4.8 \mathrm{eV}$ and the occupied molecular orbital of PEDOT is $5.1 \mathrm{eV}$. This indicates a large hole-injection barrier between the composite film and $\mathrm{Si}$ with a conduction band of $5.2 \mathrm{eV}$, thus resulting in higher $V_{\text {oc }}$ and $\mathrm{FF}$ and leading to a $40 \%$ increase in efficiency. Furthermore, as the GWF networks provides many gaps, redox electrolyte containing $40 \% \mathrm{HBr}$ and $3 \% \mathrm{Br}_{2}$ was dropped on the GWF side, forming a hybrid solar cell which consists of two types of photovoltaic systems, including a Schottky junction cell and a photoelectrochemical cell connected in parallel (see the corresponding energy diagram in the inset of Fig. 5c), cooperating with each other ${ }^{31,32}$. By this treatment, we obtain a $30 \%$ increase in efficiency (Fig. 5c).

\section{Discussion}

The easy operation in transferring is partly due to the favourable mechanical properties of the woven structure and the multilayer fishnet stacks. As the GWF is composed of GMRs, both the adhesion and friction between the two sets of GMRs (the warp and the weft) caused by the hairness of the ribbon surface play important roles in determining the fabric strength. When the fabric is being stretched in the warp direction, the weft ribbon would shrink more and intensify the friction between the two sets of GMRs. Similar to a traditional woven fabric, the clamping action of the weft on the warp would reduce the unevenness of the warp GMRs, resulting in enhanced warp ribbon strength. On the other hand, the weft GMRs might share some of the external load. The absence of visible cracking or tearing suggests high levels of physical toughness and mechanical flexibility. On the basis of a conventional theory ${ }^{33}$, the strength of the GWF has been predicated (see Fig. S8 and calculation details in Supplementary Information). Although such structures are mechanically robust, handling or otherwise manually manipulating them is difficult owing to their polycrystalline feature. As a result, similar to CVD grown graphene films, the strength of a GWF cannot be measured by direct tensile test. Practical applications still require a supporting substrate of some type (either liquid or solid).

As of the electromechanical properties of the GWF/PDMS hybrid film, the repeatable results of stretching in two different ways reveal that the GWF/PDMS resistance is sensitive to the elongation rather than the shear strain. The step-by-step crack formation in GWF upon external loading provides non-linear resistive response which is essential for highly sensitive strain sensing. Since a strain gauge always gives a linear response on 

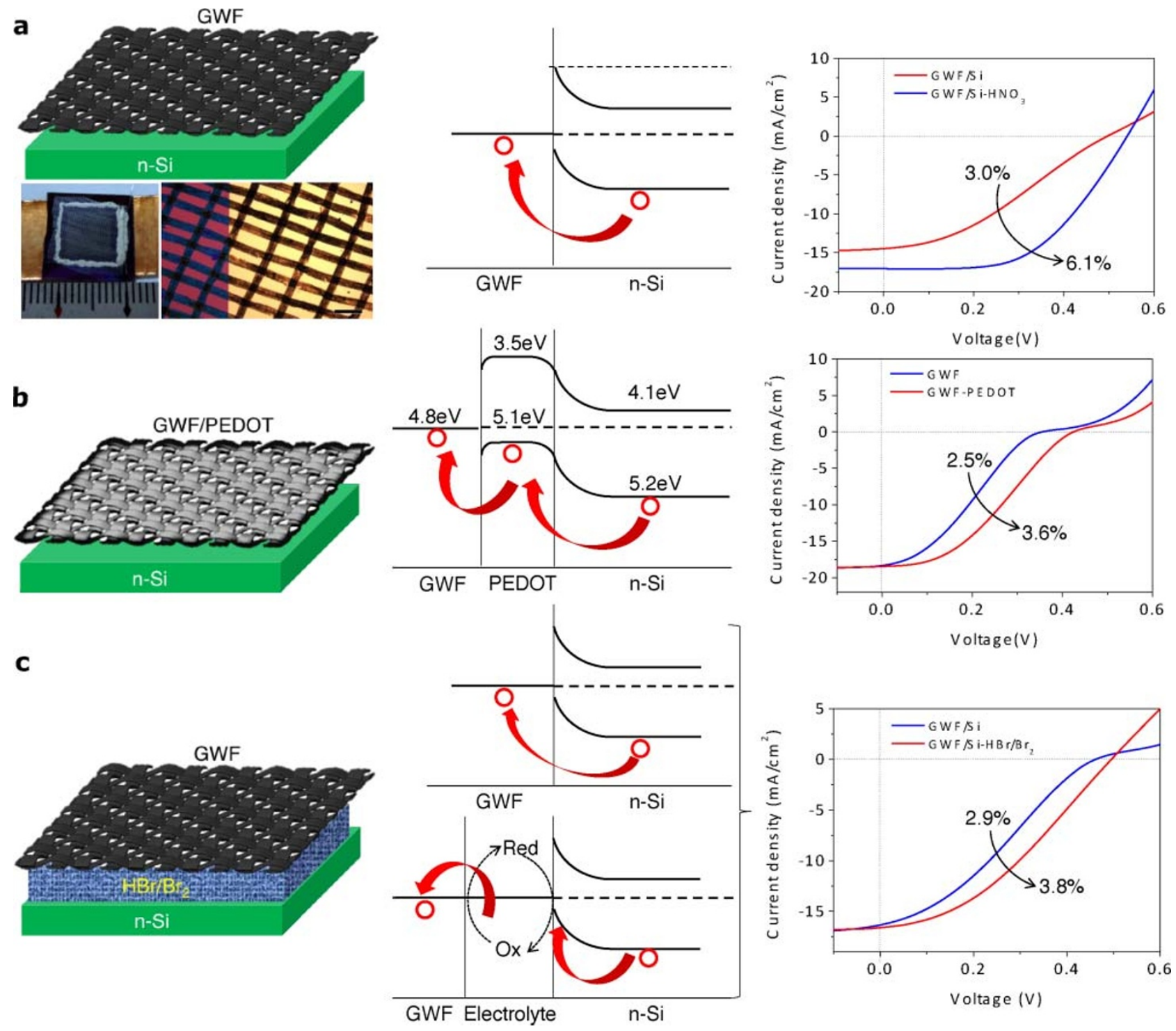

Figure 5 | GWF-based solar cells. Device schematics, energy band structure diagrams and $J$ - $V$ curves of (a) GWF/Si solar cell, (b) PEDOT filled GWF/Si solar cell and (c), hybrid Schottky and PEC GWF/Si solar cell.

elongation, the exponential response on elongation shown here might implicate a subtle metal-insulator transition ${ }^{34}$ by strain engineering due to the macroscopic motion of graphene flakes in polycrystalline GMRs, which requires further investigations to understand the underlying physics.

The greater electrical conductivity of the GWF film (as thin as $1 \mathrm{~nm}$ ) would make it possible to retain adequate lateral electrical conductivity while reducing the thickness of, or eliminating entirely, the traditional semitransparent conductive layer. The GWF film is coated conformally onto a patterned substrate to form heterojunction with the underlying semiconductor (e.g. $\mathrm{Si}$, $\mathrm{CdSe}, \mathrm{GaAs}$ with band gaps of $1.0 \sim 2.5 \mathrm{eV}$ ). As shown in the energy band diagrams (Fig. 5), our calculations indicate that carbon makes a Schottky junction with silicon ${ }^{30}$, which is favorable for producing a relatively large built-in field and charge separation. A space charge region is formed in the silicon near the interface. This indicates that the GWF film not only serves as a transparent electrode for light illumination, but also an active layer for electron/hole separation and hole transport. During this process, light absorption by the silicon produces photo-generated carriers (electron/hole pairs); ii) photogenerated holes $(\mathrm{h}+$ ) and electrons (e-) are separated and accelerated by the built-in electric field; iii) the holes are driven into the GWF film and the electrons are driven into the silicon, respectively to produce electricity. The use of GWF films in this type of solar device is a particularly attractive approach for several reasons: the extremely high surface area offers a tremendous opportunity for exciton dissociation; in addition to enhancing photovoltaic conversion efficiency, the incorporation of mesh structure can potentially improve mechanical and environmental stability.

In summary, transparent, flexible electrodes using GMRs woven in a mesh configuration are prepared by the CVD approach. The GMRs are assembled into a fabric mesh having $100 \sim 200 \mu \mathrm{m}$ spacings in orthogonally directed layouts. The GWFs are mechanically flexible because of their 2D flat morphology, with lengths greater than centimeters in some cases. The GWFs can be further optimized for conductivity and transparency through controlling of the micron-ribbon ratios and spacing. The ease of handling and the low fabrication cost have made fabrics attractive for structural and functional applications (e.g. composites and solar cells). The GWFs are compatible with plastic substrates and scalable to high throughput roll-to-roll or printing processes, thus showing the potential to 
impact future flexible electronics technology. Because of the presence of crystallographic defects like grain boundaries, the GMRs are not yet very strong, far below their ideal mechanical strength.

We are in the process of trying to develop these GWFs as a new class of flexible photovoltaic and composite materials. Our approach shows a possibility for full integration of flexible electronics within a woven smart textile. If properly tailored, hybrid electronic and optical properties of our GWFs will be utilized for future nano-optics and nano-optoelectronic engineering. The optimization and further development of this GWF technology will promise many promising applications, including heat insulating, ventilating but water and liquid proof, radiation resistant coatings, filters, masks, templates, logic circuits, TEM grids, catalyst carriers, etc.

\section{Methods}

Synthesis of GWFs. Copper meshes with different line width and spacing were used as scaffold templates for the CVD growth of GWFs. The mesh was cut into pieces of $5 \times 8 \mathrm{~cm}^{2}$ which depended on the space of the CVD reactor tube and then was ultrasonically treated with hydrochloric acid and acetone to clean their surfaces and eliminate the thin surface oxide layer. Then the mesh was heated to $1000^{\circ} \mathrm{C}$ in a CVD furnace under $\mathrm{Ar}(500 \mathrm{~mL} / \mathrm{min})$ and $\mathrm{H}_{2}(50 \mathrm{~mL} / \mathrm{min})$. After reaching $1000^{\circ} \mathrm{C}$, the $\mathrm{Ar}$ was turned down to $200 \mathrm{~mL} / \mathrm{min}$ and $\mathrm{H}_{2}$ to $20 \mathrm{~mL} / \mathrm{min}$. A small amount of $\mathrm{CH}_{4}(3 \sim 15 \mathrm{~mL} / \mathrm{min})$ was then introduced into the reactor at ambient pressure. After $10 \mathrm{~min}$ growth, the mesh was rapidly cooled down to room temperature. Like graphene grown on copper foils, GWFs from copper meshes are also polycrystalline.

Characterizations. The samples were characterized using optical microscope (OLYMPUS BX60), scanning electron microscope (SEM, LEO1530, $10 \mathrm{kV}$ ), transmission electron microscope (TEM, JEOL2010, $200 \mathrm{kV}$ ), Raman spectroscopy (Renishaw, RM2000, He-Ne laser excitation line at $514 \mathrm{~nm}$ ). To measure its transmittance and sheet resistance, the GWF was transferred to a highly polished quartz substrate. Optical transmission spectra were taken by UV, Hitachi V-3010 optical spectrometer.

GWF/polymer composites. GWF-on-PDMS hybrid films: GWF was put into $\mathrm{FeCl}_{3} /$ $\mathrm{HCl}$ mixture $(1: 1, \mathrm{~mol} / \mathrm{L})$ to dissolve the copper. Fig. S9 illustrates the etching process of a single copper wire. The lower part of the copper wire immersed in the solution was etched way firstly. During etching, the graphene film would not spread since the upper graphene is still wrapping on the copper surface, thus keeping the folding state of lower graphene. Then the upper part exposed to air gradually sank into the solution and pulled the lower part. About $2 \mathrm{~h}$ later, the copper dissolved completely, and GWF collapsed to form the planar mesh structure floating on the solution. The width of the resulting GMR approximately equals to the diameter of the copper wire. Solidified PDMS (1 mm thick) film was used to collect the GWF.

GWF-in-PDMS composites: To avoid structural failure of the GWF during copper etching, a thin layer of PDMS was used as a support to reinforce the GWF structure. The copper mesh after graphene growth was drop-coated with PDMS solution, and then baked at $60^{\circ} \mathrm{C}$ for $12 \mathrm{~h}$. After the PDMS solidified completely, the composites were put into $\mathrm{FeCl}_{3} / \mathrm{HCl}$ mixture $(1: 1, \mathrm{~mol} / \mathrm{L})$ to dissolve the copper at $80^{\circ} \mathrm{C}$. About $5 \mathrm{~h}$ later, the copper dissolved completely to give the free-standing GWF/PDMS composites.

GWF/Si solar cells. $\mathrm{n}-\mathrm{Si}(100)$ wafers (doping density: $1.5 \sim 3 \times 10^{15} / \mathrm{cm}^{3}$ ) with a $300 \mathrm{~nm} \mathrm{SiO}{ }_{2}$ layer were patterned by photolithography and wet-etching of oxide (by hydrofluoric acid solution) to make square windows $\left(0.1 \mathrm{~cm}^{2}\right)$ where $\mathrm{n}$-Si was exposed. Sputtered Ti/Au was used as the back contact. The GWF film was then transferred to the top of the patterned wafer and naturally dried to make a conformal coating with $\mathrm{n}$-Si. In the heterojunction, GWF serves as the transparent conducting window electrode, hole collector and anti-reflection layer. Similar to graphene films, GWFs impart a significant reduction of the reflectance compared to silicon (Fig. S10). The solar devices were tested with a solar simulator (Newport) under AM 1.5 condition $\left(80 \mathrm{~mW} / \mathrm{cm}^{2}\right)$. The current-voltage data were recorded using a Keithley 2602 SourceMeter. The photovoltaic performances of tested cells are summarized in Table S1.

1. Li, Y., Qian, F., Xiang, J. \& Lieber, C. M. Nanowire electronic and optoelectronic devices. Mater. Today 9, 18-27 (2006).

2. Avouris, P. \& Chen, J. Nanotube electronics and optoelectronics. Mater. Today $\mathbf{9}$, 46-54 (2006).

3. Rueckes, T., Kim, K., Joselevich, E., Tseng, G. Y., Cheung, C. L. \& Lieber, C. M. Carbon nanotube-based nonvolatile random access memory for molecular computing. Science 289, 94-97 (2000).

4. Chen, Z. H., Appenzeller, J., Lin, Y. M., Sippel-Oakley, J., Rinzler, A. G., Tang, J. Y., Wind, S. J., Solomon, P. M. \& Avouris, P. An integrated logic circuit assembled on a single carbon nanotube. Science 311, 1735-1735 (2006).
5. Yao, J., Liu, Z. W., Liu, Y. M., Wang, Y., Sun, C., Bartal, G., Stacy, A. M. \& Zhang, $\mathrm{X}$. Optical negative refraction in bulk metamaterials of nanowires. Science 321, 930-930 (2008)

6. Ju, L., Geng, B. S., Horng, J., Girit, C., Martin, M., Hao, Z., Bechtel, H. A., Liang, X. G., Zettl, A., Shen, Y. R. \& Wang, F. Graphene plasmonics for tunable terahertz metamaterials. Nat. Nanotechnol. 6, 630-634(2011).

7. Nikolaenko, A. E., De Angelis, F., Boden, S. A., Papasimakis, N., Ashburn, P., Di Fabrizio, E. \& Zheludev, N. I. Carbon nanotubes in a photonic metamaterial. Phys. Rev. Lett. 104, 153902 (2010).

8. Hamedi, M., Forchheimer, R. \& Inganas, O. Towards woven logic from organic electronic fibres. Nat. Mater. 6, 357-362 (2007).

9. Lin, D., Wu, H. \& Pan, W. Photoswitches and memories assembled by electrospinning aluminum-doped zinc oxide single nanowires. Adv. Mater. 19, 3968-3972 (2007).

10. Teo, W. E. \& Ramakrishna, S. Electrospun nanofibers as a platform for multifunctional, hierarchically organized nanocomposite. Compos. Sci. Technol. 69, 1804-1817 (2009)

11. Cherenack, K., Zysset, C., Kinkeldei, T., Munzenrieder, N. \& Troster, G. Woven electronic fibers with sensing and display functions for smart textiles. Adv. Mater. 22, 5178-5182 (2010).

12. Kylberg, W., de Castro, F. A., Chabrecek, P., Sonderegger, U., Chu, B. T. T., Nuesch, F. \& Hany, R. Woven electrodes for flexible organic photovoltaic Cells. Adv. Mater. 23, 1015-1019 (2011).

13. Hong, I. H., Liao, Y. C. \& Yen, S. C. Self-organization of a highly integrated silicon nanowire network on a $\mathrm{Si}(110)-16 \times 2$ surface by controlling domain growth. $A d v$. Funct. Mater. 19, 3389-3395 (2009).

14. Liu, L. Q., Ma, W. J. \& Zhang, Z. Macroscopic carbon nanotube assemblies: preparation, properties, and potential applications. Small 7, 1504-1520 (2011).

15. Liu, K., Sun, Y. H., Liu, P., Lin, X. Y., Fan, S. S. \& Jiang, K. L. Cross-stacked superaligned carbon nanotube films for transparent and stretchable conductors. Adv. Funct. Mater. 21, 2721-2728 (2011).

16. Chen, Z. P., Ren, W. C., Gao, L. B., Liu, B. L., Pei, S. F. \& Cheng, H. M. Threedimensional flexible and conductive interconnected graphene networks grown by chemical vapour deposition. Nat. Mater. 10, 424-428 (2011).

17. Xu, Y. X., Sheng, K. X., Li, C. \& Shi, G. Q. Self-assembled graphene hydrogel via a one-step hydrothermal process. ACS Nano 4, 4324-4330 (2010).

18. Bai, J. W., Zhong, X., Jiang, S., Huang, Y. \& Duan, X. F. Graphene nanomesh. Nat. Nanotechnol. 5, 190-194 (2010).

19. Song, L., Ci, L. J., Gao, W. \& Ajayan, P. M. Transfer printing of graphene using gold film. ACS Nano 3, 1353-1356 (2009).

20. Kim, T. Y., Kwon, S. W., Park, S. J., Yoon, D. H., Suh, K. S. \& Yang, W. S. Selforganized graphene patterns. Adv. Mater. 23, 2734-2738 (2011).

21. Horiuchi, S., Gotou, T., Fuijwara, M., Sotoaka, R., Hirata, M., Kimoto, K., Asaka, T., Yokosawa, T., Matsui, Y., Watanabe, K. \& Sekita, M. Carbon nanofilm with a new structure and property. Jpn. J. Appl. Phys. Part 2 42, L1073-L1076 (2003).

22. Ferrari, A. C., Meyer, J. C., Scardaci, V., Casiraghi, C., Lazzeri, M., Mauri, F., Piscanec, S., Jiang, D., Novoselov, K. S., Roth, S. \& Geim, A. K. Raman spectrum of graphene and graphene layers. Phys. Rev. Lett. 97, 187401 (2006).

23. Li, X. S., Magnuson, C. W., Venugopal, A., An, J. H., Suk, J. W., Han, B. Y. Borysiak, M., Cai, W. W., Velamakanni, A., Zhu, Y. W., Fu, L. F., Vogel, E. M., Voelkl, E., Colombo, L. \& Ruoff, R. S. Graphene films with large domain size by a two-step chemical vapor deposition process. Nano Lett. 10, 4328-4334 (2010).

24. Bae, S., Kim, H., Lee, Y., Xu, X. F., Park, J. S., Zheng, Y., Balakrishnan, J., Lei, T., Kim, H. R., Song, Y. I., Kim, Y. J., Kim, K. S., Ozyilmaz, B., Ahn, J. H., Hong, B. H. \& Iijima, S. Roll-to-roll production of 30-inch graphene films for transparent electrodes. Nat. Nanotechnol. 5, 574-578 (2010).

25. Kim, K. S., Zhao, Y., Jang, H., Lee, S. Y., Kim, J. M., Kim, K. S., Ahn, J. H., Kim, P., Choi, J. Y. \& Hong, B. H. Large-scale pattern growth of graphene films for stretchable transparent electrodes. Nature 457, 706-710 (2009).

26. Lipomi, D. J., Vosgueritchian, M., Tee, B. C. K., Hellstrom, S. L., Lee, J. A., Fox, C. H. \& Bao, Z. N. Skin-like pressure and strain sensors based on transparent elastic films of carbon nanotubes. Nat. Nanotechnol. 6, 788-792 (2011).

27. Bonaccorso, F., Sun, Z., Hasan, T. \& Ferrari, A. C. Graphene photonics and optoelectronics. Nat. Photonics 4, 611-622 (2010).

28. Li, X. M., Zhu, H. W., Wang, K. L., Cao, A. Y., Wei, J. Q., Li, C. Y., Jia, Y., Li, Z., Li, X. \& Wu, D. H. Graphene-on-silicon Schottky junction solar cells. Adv. Mater. 22, 2743-2748 (2010).

29. Li, Z., Zhu, H. W., Xie, D., Wang, K. L., Cao, A. Y., Wei, J. Q., Li, X. A., Fan, L. L. \& $\mathrm{Wu}, \mathrm{D}$. H. Flame synthesis of few-layered graphene/graphite films. Chem. Commun. 47, 3520-3522 (2011).

30. Jia, Y., Cao, A. Y., Bai, X., Li, Z., Zhang, L. H., Guo, N., Wei, J. Q., Wang, K. L., Zhu, H. W., Wu, D. H. \& Ajayan, P. M. Achieving high efficiency silicon-carbon nanotube heterojunction solar cells by acid doping. Nano Lett. 11, 1901-1905 (2011).

31. Shu, Q. K., Wei, J. Q., Wang, K. L., Zhu, H. W., Li, Z., Jia, Y., Gui, X. C., Guo, N., Li, X. M., Ma, C. R. \& Wu, D. H. Hybrid heterojunction and photoelectrochemistry solar cell based on silicon nanowires and double-walled carbon nanotubes. Nano Lett. 9, 4338-4342 (2009). 
32. Shu, Q. K., Wei, J. Q., Wang, K. L., Song, S. A., Guo, N., Jia, Y., Li, Z., Xu, Y., Cao, A. Y., Zhu, H. W. \& Wu, D. H. Efficient energy conversion of nanotube/nanowirebased solar cells. Chem. Commun. 46, 5533-5535 (2010).

33. Pan, N. Analysis of woven fabric strengths: Prediction of fabric strength under uniaxial and biaxial extensions. Compos. Sci. Technol. 56, 311-327 (1996).

34. Imada, M., Fujimori, A. \& Tokura, Y. Metal-insulator transitions. Rev. Mod. Phys. 70, 1039-1263 (1998).

\section{Acknowledgments}

This work was supported by the National Program on Key Basic Research Project (973 Program, 2011CB013000), the National Science Foundation of China (50972067), and the Beijing Natural Science Foundation (2122027).

\section{Author contributions}

H.W.Z. and X.L. conceived and designed the experiments. X.L., P.Z.S., L.L.F. performed the experiments. All authors interpreted the results. H.W.Z. and X.L. co-wrote the manuscript.

\section{Additional information}

Supplementary information accompanies this paper at http://www.nature.com/ scientificreports

Competing financial interests: The authors declare no competing financial interests.

License: This work is licensed under a Creative Commons

Attribution-NonCommercial-ShareAlike 3.0 Unported License. To view a copy of this license, visit http://creativecommons.org/licenses/by-nc-sa/3.0/

How to cite this article: Li, X. et al. Multifunctional graphene woven fabrics. Sci. Rep. 2, 395; DOI:10.1038/srep00395 (2012). 